\title{
Penetrating eye injury from a crayfish antenna
}

Khoi A Tran MMed, MB BS, BPharm, Ophthalmology Registrar

Matthew Green MSC, MB BS Ophthalmology Registrar

\section{Jayne Camuglia BSc, MB Ophthalmology Registrar \\ Stephen O'Hagan MB BS, FRANZCO Ophthalmologist \\ 1 Department of Ophthalmology, \\ Sydney Eye Hospital, Sydney, NSW. \\ 2 Cairns Base Hospital, Cairns, QLD. \\ k_tran_@mac.com}

doi: 10.5694/mjal0.10794

\section{This is the first reported case of eye injury caused by a crayfish antenna. A 20-year-old male crayfish diver sustained a scleral penetrating injury that led to a subconjunctival abscess. The foreign body was histologically similar to a crayfish antenna.}

\section{Clinical record}

A 20-year-old man who worked as a crayfish diver presented with a unilateral red watery right eye, initially developed while at sea. He was unable to continue diving, mainly due to photophobia, and on reaching land he was transferred to our hospital by aeroplane. He had no other medical history, regular medications or known allergies. Seven days before presentation, he had a sore ear on the right side with pain radiating down the right hand side of his neck and discharge from his nose; however, he denied any trauma to the right eye.

At first presentation to the ophthalmologist, the patient's vision was $6 / 36$ in the right eye and 6/6 in the left eye. The vision in his right eye improved to $6 / 18$ with pinhole. He had peripheral corneal superficial punctate keratitis, $3+$ cells in the anterior chamber, and an elevation of the conjunctiva, inferior to the cornea, $4.5 \mathrm{~mm}$ in diameter. Examination of his retina was unremarkable, and he had a small nasal pterygium. Examination of the left eye was unremarkable. The intraocular pressure was $12 \mathrm{mmHg}$ in both eyes. He was provisionally diagnosed with right eye acute anterior uveitis and treated with topical combined prednisolone acetate plus phenylephrine hydrochloride and 2\% homatropine hydrobromide for mydriasis.

At a scheduled review 2 days later, the condition of the patient's right eye had deteriorated. He had developed an anteriorly discharging subconjunctival abscess. A combined duplex B-scan ultrasound showed a hypoechoic, hypervascular soft tissue mass in, or external to, the sclera and inferomedial to the iris. The mass was $7 \mathrm{~mm}$ deep and protruded into the globe.

The patient was immediately taken to the operating theatre for excision and exploration of the subconjunctival abscess. Intraoperative examination with a three-mirror gonioscope lens before excision showed an unidentifiable membranous foreign body in the anterior chamber. The foreign body was thought to be a parasite due to its longitudinal appearance.

The subconjunctival abscess was excised and explored. A tract from the subconjunctival space through the full scleral thickness and into the suprachoroidal space was explored, and the foreign body in the anterior chamber was removed. The scleral defect was covered with a conjunctival graft using cardinal and continuous sutures, and the overlying conjunctival defect was repaired with a conjunctival rotation.

After surgery, the patient was treated with intravenous ceftriaxone and topical ofloxacin $(3 \mathrm{mg} / \mathrm{mL})$, prednisolone acetate plus phenylephrine hydrochloride and homatropine hydrobromide eye drops, oral analgesia, and three doses of albendazole $(400 \mathrm{mg})$.

Two days after surgery, the patient's condition had improved considerably. His vision had improved to 6/6 with pinhole and the anterior chamber cells had improved to $2+$ cells. He was discharged home on topical prednisolone acetate plus phenylephrine hydrochloride, ofloxacin and homatropine hydrobromide eye drops, and oral prednisolone.

Histological results of the initial surgical specimen showed a small foreign body several microns in length intimately associated with deeply placed active inflammation (Box 1). There was fibroproliferative activity and an infiltrate of inflammatory cells; these were associated with tissue necrosis and microabscess formation. Culture of a preoperative conjunctival swab was negative for microbial growth.

Three days after discharge, the patient returned to hospital with a recurrence of the subconjunctival abscess, for which he underwent emergency excision and drainage. The previous conjunctival graft was found to be necrotic and was removed. A thin fibrovascular membrane covering the scleral defect remained. A swab taken from the abscess was later positive for methicillin-resistant Staphylococcus aureus, which was successfully treated with intravenous vancomycin, oral clindamycin and topical chloramphenicol.

At 4 weeks' follow-up, the patient's unaided visual acuity in his right eye had stabilised to $6 / 6$ and the intraocular pressure was $7-8 \mathrm{mmHg}$. The conjunctival flap had dehisced, but there was a steady increase in the thickness of the fibrovascular covering of the scleral perforation.

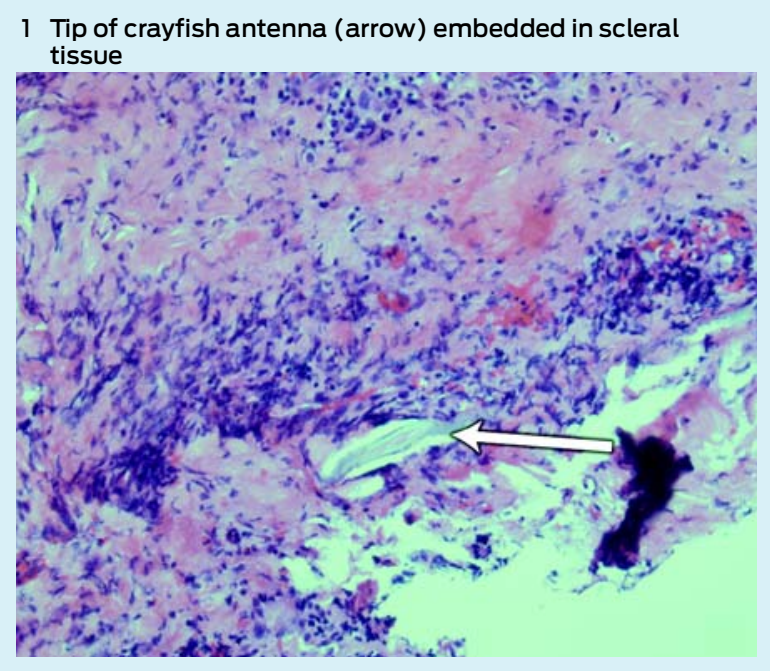


The original foreign body extracted during surgery was compared with a specimen of crayfish antennae and was found to be similar to the very outer layer $(1-2 \mu \mathrm{m})$ of the specimen (Box 2). It most likely became lodged while the patient was handling crayfish or cleaning the crayfish tank without eye protection, which he was doing extensively during the morning that the red eye developed. The crayfish caught in this area are of several different types, although the Panulirus ornatus (tropical rock lobster) make up 95\% of the local population. ${ }^{1}$

\section{Discussion}

Penetrating eye injuries (PEIs) are serious and can result in blindness, either from direct trauma to ocular structures or secondary infection and inflammation. Given the significant consequences of PEIs, early diagnosis and management is imperative to improve outcomes. Despite the vast majority of PEIs being obvious through history of trauma, some are less evident to both patient and clinician. We report a case of delayed diagnosis of PEI due to a unique mechanism.

A population-based cross-sectional study of eye injuries in Australia found that they were more common among men, people living in rural areas and tradespeople, and that the workplace was the most common location where the injury occurred. ${ }^{2}$ A review of 109 penetrated or ruptured globes over 4 years in Adelaide identified that $80 \%$ of patients were men; the most common cause of injury was hammering metal; and the final visual acuity was $6 / 12$ or better in $40 \%$ of patients, and no perception of light in $26 \%{ }^{3}$ Another group found that of 6308 patients treated at their hospital over 12 months, 6\% were admitted for severe ocular trauma and most of these were young men. ${ }^{4}$

Workplace-related injuries accounted for $44 \%$ of all ocular injuries and $19 \%$ of cases of severe eye trauma. ${ }^{4}$ The annual medical costs for eye injuries have been estimated at \$155 million for the projected 116000 cases nationwide. ${ }^{4}$ In 2006, a review of ocular trauma over 7 years at Cairns Base Hospital estimated the incidence of open globe injuries to be 3.7 per 100000 population. ${ }^{5}$ An international study of PEIs in rural areas found that $16 \%$ were work-related. ${ }^{6}$ Most injuries were related to maintenance or repair work, wood chopping or machine use. A small number were the result of cow butting. At the final review, $64 \%$ of these eyes were blind. $^{6}$

Crayfish antenna penetration is a mechanism that has not previously been reported. Thorns of plants have been previously reported to cause PEIs in children, but the most common aquatic-related PEI is with fish hooks. ${ }^{7,8}$ Appropri-

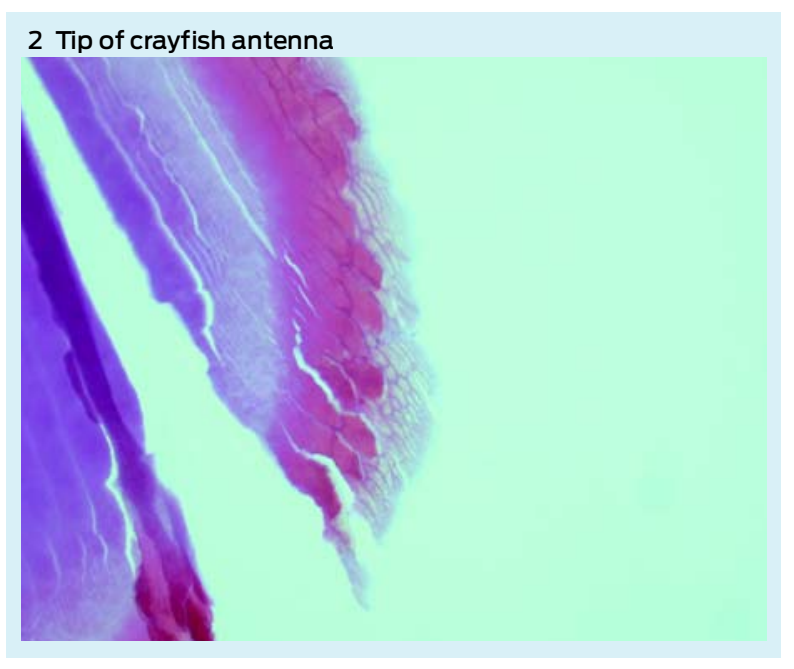

ate preventive measures, such as use of protective eyewear and implementation of education programs, could be effective in decreasing the incidence of these aquatic-related ocular traumas.

Our case highlights the risk of PEIs for young men while at work. It demonstrates the possibility of a small organic foreign body associated with a large inflammatory reaction and a full-thickness scleral penetration that could have resulted in blindness. For clinicians, our case emphasises the importance of early suspicion of ocular trauma in high-risk patients with red eyes, particularly if they have an unexpected lack of response or deterioration to treatment despite an initially negative history of trauma.

Competing interests: No relevant disclosures.

Received 21 Jul 2010, accepted 10 Oct 2011.

1 Fisheries of Queensland's East Coast CRC Reef Research Centre. Tropical rock lobster. 2005. http://www.reef.crc.org.au/research/fishing_fisheries/ statusfisheries/harvestlobster.htm (accessed Nov 2011).

2 McCarty CA, Fu CL, Taylor HR. Epidemiology of ocular trauma in Australia. Ophthalmology 1999; 106: 1847-1852.

3 Casson RJ, Walker JC, Newland HS. Four-year review of open eye injuries at the Royal Adelaide Hospital. Clin Experiment Ophthalmol 2002; 30: 15-18.

4 Fong LP. Eye injuries in Victoria, Australia. Med J Aust 1995; 162: 64-68.

5 Smith AR, O'Hagan SB, Gole GA. Epidemiology of open and closed globe trauma presenting to Cairns Base Hospital, Queensland. Clin Experiment Ophthalmol 2006; 34: 252-259.

6 Mackiewicz J, Machowicz-Matejko E, Saaga-Pylak M, et al. Work-related, penetrating eye injuries in rural environments. Ann Agric Environ Med 2005; 12: $27-29$.

7 Blomdahl S, Norell S. Perforating eye injury in the Stockholm population. An epidemiological study. Acta Ophthalmol (Copenh) 1984; 62: 378-390.

8 Knox FA, Chan WC, McAvoy CE, et al. Penetrating ocular injuries from fishhooks. Int Ophthalmol 2004; 25: 291-294. 